\title{
Research Article \\ On the Convergence of Iterative Processes for Generalized Strongly Asymptotically $\phi$-Pseudocontractive Mappings in Banach Spaces
}

\author{
Vittorio Colao \\ Dipartimento di Matematica, Universitá della Calabria, 87036 Arcavacata di Rende (CS), Italy \\ Correspondence should be addressed to Vittorio Colao, colao@mat.unical.it
}

Received 5 October 2011; Accepted 11 October 2011

Academic Editor: Yonghong Yao

Copyright (c) 2012 Vittorio Colao. This is an open access article distributed under the Creative Commons Attribution License, which permits unrestricted use, distribution, and reproduction in any medium, provided the original work is properly cited.

We prove the equivalence and the strong convergence of iterative processes involving generalized strongly asymptotically $\phi$-pseudocontractive mappings in uniformly smooth Banach spaces.

\section{Introduction}

Throughout this paper, we assume that $X$ is a uniformly convex Banach space and $X^{*}$ is the dual space of $X$. Let $J$ denote the normalized duality mapping form $X$ into $2^{X^{*}}$ given by $J(x)=\left\{f \in X^{*}:\langle x, f\rangle=\|x\|^{2}=\|f\|^{2}\right\}$ for all $x \in X$, where $\langle\cdot, \cdot\rangle$ denotes the generalized duality pairing. It is well known that if $X$ is uniformly smooth, then $J$ is single valued and is norm to norm uniformly continuous on any bounded subset of $X$. In the sequel, we will denote the single valued duality mapping by $j$.

In 1967, Browder [1] and Kato [2], independently, introduced accretive operators (see, for details, Chidume [3]). Their interest is connected with the existence of results in the theory of nonlinear equations of evolution in Banach spaces.

In 1972, Goebel and Kirk [4] introduced the class of asymptotically nonexpansive mappings as follows.

Definition 1.1. Let $K$ be a subset of a Banach space $X$. A mapping $T: K \rightarrow K$ is said to be asymptotically nonexpansive if for each $x, y \in K$

$$
\left\|T^{n} x-T^{n} y\right\| \leq k_{n}\|x-y\|
$$

where $\left\{k_{n}\right\}_{n} \subset[1, \infty)$ is a sequence of real numbers converging to 1 . 
This class is more general than the class of nonexpansive mappings as the following example clearly shows.

Example 1.2 (see [4]). If $B$ is the unit ball of $l^{2}$ and $T: B \rightarrow B$ is defined as

$$
T\left(x_{1}, x_{2}, \ldots\right)=\left(0, x_{1}^{2}, a_{2} x_{2}, a_{3} x_{3}, \ldots\right)
$$

where $\left\{a_{i}\right\}_{i \in \mathbb{N}} \subset(0,1)$ is such that $\prod_{i=2}^{\infty} a_{i}=1 / 2$, it satisfies.

$$
\|T x-T y\| \leq 2\|x-y\|, \quad\left\|T^{n} x-T^{n} y\right\| \leq 2 \prod_{j=2}^{n} a_{i}\|x-y\| .
$$

In 1974, Deimling [5], studying the zeros of accretive operators, introduced the class of $\varphi$-strongly accretive operators.

Definition 1.3. An operator $A$ defined on a subset $K$ of a Banach space $X$ is said, $\varphi$-strongly accretive if

$$
\langle A x-A y, j(x-y)\rangle \geq \varphi(\|x-y\|)\|x-y\|
$$

where $\varphi: \mathbb{R}^{+} \rightarrow \mathbb{R}^{+}$is a strictly increasing function such that $\varphi(0)=0$.

Note that in the special case in which $\varphi(t)=k t, k \in(0,1)$, we obtain a strongly accretive operator.

Osilike [6], among the others, proved that $A x=x-(x /(x+1))$ in $\mathbb{R}^{+}$is $\varphi$-strongly accretive where $\varphi(t)=\left(t^{2} /(1+t)\right)$ but not strongly accretive.

Since an operator $A$ is a strongly accretive operator if and only if $(I-A)$ is a strongly pseudocontractive mapping (i.e., $\left.\langle(I-A) x-(I-A) y, j(x-y)\rangle \leq k\|x-y\|^{2}, k<1\right)$, taking in to account Definition 1.3, it is natural to study the class of $\varphi$-pseudocontractive mappings, that is, the maps such that

$$
\langle T x-T y, j(x-y)\rangle \leq\|x-y\|^{2}-\varphi(\|x-y\|)\|x-y\|
$$

where $\varphi: \mathbb{R}^{+} \rightarrow \mathbb{R}^{+}$is a strictly increasing function such that $\varphi(0)=0$. Of course, the set of fixed points for these mappings contains, at most, only one point.

Recently, has been also studied the following class of maps.

Definition 1.4. A mapping $T$ is a generalized $\phi$-strongly pseudocontractive mapping if

$$
\langle T x-T y, j(x-y)\rangle \leq\|x-y\|^{2}-\phi(\|x-y\|),
$$

where $\phi: \mathbb{R}^{+} \rightarrow \mathbb{R}^{+}$is a strictly increasing function such that $\phi(0)=0$.

Choosing $\phi(t)=\varphi(t) t$, we obtain Definition 1.3. In [7], Xiang remarked that it is a open problem if every generalized $\phi$-strongly pseudocontractive mapping is $\varphi$-pseudocontractive 
mapping. In the same paper, Xiang obtained a fixed-point theorem for continuous and generalized $\phi$-strongly pseudocontractive mappings in the setting of the Banach spaces.

In 1991, Schu [8] introduced the class of asymptotically pseudocontractive mappings.

Definition 1.5 (see [8]). Let $X$ be a normed space, $K \subset X$ and $\left\{k_{n}\right\}_{n} \subset[1, \infty)$. A mapping $T: K \rightarrow K$ is said to be asymptotically pseudocontractive with the sequence $\left\{k_{n}\right\}_{n}$ if and only if $\lim _{n \rightarrow \infty} k_{n}=1$, and for all $n \in \mathbb{N}$ and all $x, y \in K$, there exists $j(x-y) \in J(x-y)$ such that

$$
\left\langle T^{n} x-T^{n} y, j(x-y)\right\rangle \leq k_{n}\|x-y\|^{2},
$$

where $J$ is the normalized duality mapping.

Obviously every asymptotically nonexpansive mapping is asymptotically pseudocontractive, but the converse is not valid; it is well known that $T:[0,1] \rightarrow[0,1]$ defined by $T x=\left(1-x^{2 / 3}\right)^{3 / 2}$ is not Lipschitz but asymptotically pseudocontractive [9].

In [8], Schu proved the following.

Theorem 1.6 (see [8]). Let $H$ be a Hilbert space and $A \subset H$ closed and convex; $L>0$; $T: A \rightarrow$ A completely continuous, uniformly L-Lipschitzian, and asymptotically pseudocontractive with sequence $\left\{k_{n}\right\}_{n} \in[1, \infty) ; q_{n}:=2 k_{n}-1$ for all $n \in \mathbb{N} ; \sum_{n}\left(q_{n}^{2}-1\right)<\infty ;\left\{\alpha_{n}\right\}_{n},\left\{\beta_{n}\right\}_{n} \in[0,1]$; $\epsilon \leq \alpha_{n} \leq \beta_{n} \leq b$ for all $n \in \mathbb{N}$, some $\epsilon>0$ and some $b \in\left(0, L^{-2}\left[\sqrt{1+L^{2}}-1\right]\right) ; x_{1} \in A$; for all $n \in \mathbb{N}$, define

$$
\begin{aligned}
z_{n} & :=\beta_{n} T^{n}\left(x_{n}\right)+\left(1-\beta_{n}\right) x_{n}, \\
x_{n+1} & :=\alpha_{n} T^{n}\left(z_{n}\right)+\left(1-\alpha_{n}\right) x_{n},
\end{aligned}
$$

then $\left\{x_{n}\right\}_{n}$ converges strongly to some fixed point of $T$.

Until 2009, no results on fixed-point theorems for asymptotically pseudocontractive mappings have been proved. First, Zhou in [10] completed this lack in the setting of Hilbert spaces proving a fixed-point theorem for an asymptotically pseudocontractive mapping that is also uniformly $L$-Lipschitzian and uniformly asymptotically regular and that the set of fixed points of $T$ is closed and convex. Moreover, Zhou proved the strong convergence of a CQ-iterative method involving this kind of mappings.

In this paper, our attention is on the class of the generalized strongly asymptotically $\phi$ pseudocontraction defined as follows.

Definition 1.7. If $X$ is a Banach space and $K$ is a subset of $X$, a mapping $T: K \rightarrow K$ is said to be a generalized asymptotically $\phi$-strongly pseudocontraction if

$$
\left\langle T^{n} x-T^{n} y, j(x-y)\right\rangle \leq k_{n}\|x-y\|^{2}-\phi(\|x-y\|),
$$

where $\left\{k_{n}\right\}_{n} \subset[1, \infty)$ is converging to one and $\phi:[0, \infty) \rightarrow[0, \infty)$ is strictly increasing and such that $\phi(0)=0$. 
One can note that

(i) if $T$ has fixed points, then it is unique. In fact, if $x, z$ are fixed points for $T$, then for every $n \in \mathbb{N}$,

$$
\|x-z\|^{2}=\left\langle T^{n} x-T^{n} z, j(x-z)\right\rangle \leq k_{n}\|x-z\|^{2}-\phi(\|x-z\|),
$$

so passing $n$ to $+\infty$, it results that

$$
\|x-z\|^{2} \leq\|x-z\|^{2}-\phi(\|x-z\|) \Longrightarrow-\phi(\|x-z\|) \geq 0 .
$$

Since $\phi:[0, \infty) \rightarrow[0, \infty)$ is strictly increasing and $\phi(0)=0$, then $x=z$.

(ii) the mapping $T x=x /(x+1)$, where $x \in[0,1]$, is generalized asymptotically strongly $\phi$-pseudocontraction with $k_{n}=1$, for all $n \in \mathbb{N}$ and $\phi(t)=s^{3} /(1+s)$. However, $T$ is not strongly pseudocontractive; see [6].

We study the equivalence between three kinds of iterative methods involving the generalized asymptotically strongly $\phi$-pseudocontractions.

Moreover, we prove that these methods are equivalent and strongly convergent to the unique fixed point of the generalized strongly asymptotically $\phi$-pseudocontraction $T$, under suitable hypotheses.

We will briefly introduce some of the results in the same line of ours. In 2001, [11] Chidume and Osilike proved the strong convergence of the iterative method

$$
\begin{aligned}
& y_{n}=a_{n} x_{n}+b_{n} S x_{n}+c_{n} u_{n}, \\
& x_{n+1}=a_{n}^{\prime} x_{n}+b_{n}^{\prime} S y_{n} c_{n}^{\prime} v_{n},
\end{aligned}
$$

where $a_{n}+b_{n}+c_{n}=a_{n}^{\prime}+b_{n}^{\prime}+c_{n}^{\prime}=1, S x=x-T x+f$ ( $T$ a $\phi$-strongly accretive operator $)$, and $f \in X$, to a solution of the equation $T x=f$.

In 2003, Chidume and Zegeye [12] studied the following iterative method:

$$
x_{n+1}=\left(1-\lambda_{n}\right) x_{n}+\lambda_{n} T x_{n}-\lambda_{n} \theta_{n}\left(x_{n}-x_{1}\right)
$$

where $T$ is a Lipschitzian pseudocontractive map with fixed points. The authors proved the strong convergence of the method to a fixed point of $T$ under suitable hypotheses on the control sequences $\left(\theta_{n}\right)_{n},\left(\lambda_{n}\right)_{n}$.

Taking in to account Chidume and Zegeye [12] and Chang [13], we introduce the modified Mann and Ishikawa iterative processes as follows: for any given $x_{0} \in X$, the sequence $\left\{x_{n}\right\}_{n}$ is defined by

$$
\begin{aligned}
& y_{n}=\left(1-\beta_{n}\right) x_{n}+\beta_{n} T^{n} x_{n}-\delta_{n}\left(x_{n}-v_{n}\right), \\
& x_{n+1}=\left(1-\alpha_{n}\right) x_{n}+\alpha_{n} T^{n} y_{n}-\gamma_{n}\left(x_{n}-u_{n}\right), \quad n \geq 0,
\end{aligned}
$$

where $\left\{\alpha_{n}\right\}_{n},\left\{\gamma_{n}\right\}_{n},\left\{\beta_{n}\right\}_{n}$, and $\left\{\delta_{n}\right\}_{n}$ are four sequences in $(0,1)$ satisfying the conditions $\alpha_{n}+\gamma_{n} \leq 1$ and $\beta_{n}+\delta_{n} \leq 1$ for all $n \geq 0$. 
In particular, if $\beta_{n}=\delta_{n}=0$ for all $n \geq 0$, we can define a sequence $\left\{z_{n}\right\}_{n}$ by

$$
\begin{aligned}
& z_{0} \in X, \\
& z_{n+1}=\left(1-\alpha_{n}\right) z_{n}+\alpha_{n} T^{n} z_{n}-\gamma_{n}\left(z_{n}-w_{n}\right), \quad n \geq 0,
\end{aligned}
$$

which is called the modified Mann iteration sequence.

We also introduce an implicit iterative process as follows:

$$
z_{n}^{\prime}=\left(1-\alpha_{n}\right) z_{n-1}^{\prime}+\alpha_{n} T^{n} z_{n}^{\prime}-\gamma_{n}\left(z_{n-1}^{\prime}-w_{n}^{\prime}\right), \quad n \geq 1,
$$

where $\left\{\alpha_{n}\right\}_{n},\left\{\gamma_{n}\right\}_{n}$ are two real sequences in [0,1] satisfying $\alpha_{n}+\gamma_{n} \leq 1$ and $\alpha_{n} k_{n}<1$ for all $n \geq 1,\left\{w_{n}^{\prime}\right\}_{n}$ is a sequence in $X$, and $z_{0}^{\prime}$ is an initial point.

The algorithm is well defined. Indeed, if $T$ is a asymptotically strongly $\phi$-pseudocontraction, one can observe that, for every fixed $n$, the mapping $S_{n}$ defined by $S_{n} x$ := $\left(1-\alpha_{n}-\gamma_{n}\right) z_{n-1}+\alpha_{n} T^{n} x+\gamma w_{n}$ is such that

$$
\left\langle S_{n} x-S_{n} y, j(x-y)\right\rangle=\left\langle T^{n} x-T^{n} y, j(x-y)\right\rangle \leq \alpha_{n} k_{n}\|x-y\|^{2},
$$

that is, $S_{n}$ is a strongly pseudocontraction, for every fixed $n$, then (see Theorem 13.1 in [14]) there exists a unique fixed point of $S_{n}$ for each $n$.

These kind of iterative processes (also called by Chang iterative processes with errors) have been developed in [15-18], while equivalence theorem for Mann and Ishikawa methods has been studied, in $[19,20]$, among the others.

In [21], Huang established equivalences between convergence of the modified Mann iteration process with errors (1.15) and convergence of modified Ishikawa iteration process with errors (1.14) for strongly successively $\phi$-pseudocontractive mappings in uniformly smooth Banach space.

In the next section, we prove that, in the setting of the uniformly smooth Banach space, if $T$ is an asymptotically strongly $\phi$-pseudocontraction, not only (1.14) and (1.15) are equivalent but also (1.16) is equivalent to the others. Moreover, we prove also that (1.14), (1.15), and (1.16) strongly converge to the unique fixed point of $T$, if it exists.

\section{Preliminaries}

We recall some definitions and conclusions.

Definition 2.1. $X$ is said to be a uniformly smooth Banach space if the smooth module of $X$

$$
\rho_{X}(t)=\sup \left\{\frac{1}{2}(\|x-y\|+\|x+y\|)-1:\|x\| \leq 1,\|y\| \leq t\right\}
$$

satisfies $\lim _{t \rightarrow 0} \rho_{X}(t) / t=0$. 
Lemma 2.2 (see [22]). Let $X$ be a Banach space, and let $j: X \rightarrow 2^{X^{*}}$ be the normalized duality mapping, then for any $x, y \in X$, one has

$$
\|x+y\|^{2} \leq\|x\|^{2}+2\langle y, j(x+y)\rangle, \quad \forall j(x+y) \in J(x+y) .
$$

The next lemma is one of the main tools for our proofs.

Lemma 2.3 (see [21]). Let $\phi:[0, \infty) \rightarrow[0, \infty)$ be a strictly increasing function with $\phi(0)=0$, and let $\left\{a_{n}\right\}_{n},\left\{b_{n}\right\}_{n},\left\{c_{n}\right\}_{n}$, and $\left\{e_{n}\right\}_{n}$ be nonnegative real sequences such that

$$
\lim _{n \rightarrow \infty} b_{n}=0, \quad c_{n}=o\left(b_{n}\right), \quad \sum_{n=1}^{\infty} b_{n}=\infty, \quad \lim _{n \rightarrow \infty} e_{n}=0 .
$$

Suppose that there exists an integer $N_{1}>0$ such that

$$
a_{n+1}^{2} \leq a_{n}^{2}-2 b_{n} \phi\left(\left|a_{n+1}-e_{n}\right|\right)+c_{n}, \quad \forall n \geq N_{1},
$$

then $\lim _{n \rightarrow \infty} a_{n}=0$.

Proof. The proof is the same as in [21], but we substitute $\left(a_{n+1}-e_{n}\right)$ with $\left|a_{n+1}-e_{n}\right|$, in (2.4).

Lemma 2.4 (see [23]). Let $\left\{s_{n}\right\}_{n^{\prime}}\left\{c_{n}\right\}_{n} \subset \mathbb{R}_{+},\left\{a_{n}\right\}_{n} \subset(0,1)$, and $\left\{b_{n}\right\}_{n} \subset \mathbb{R}$ be sequences such that

$$
s_{n+1} \leq\left(1-a_{n}\right) s_{n}+b_{n}+c_{n}
$$

for all $n \geq 0$. Assume that $\sum_{n}\left|c_{n}\right|<\infty$, then the following results hold:

(1) if $b_{n} \leq \beta a_{n}$ (where $\beta \geq 0$ ), then $\left\{s_{n}\right\}_{n}$ is a bounded sequence;

(2) if one has $\sum_{n} a_{n}=\infty$ and $\lim \sup _{n} b_{n} / a_{n} \leq 0$, then $s_{n} \rightarrow 0$ as $n \rightarrow \infty$.

Remark 2.5. If in Lemma 2.3 choosing $e_{n}=0$, for all $n, \phi(t)=k t^{2}(k<1)$, then the inequality (2.4) becomes

$$
\begin{aligned}
a_{n+1}^{2} & \leq a_{n}^{2}-2 b_{n} k a_{n+1}^{2}+c_{n} \Longrightarrow \\
a_{n+1}^{2} & \leq \frac{1}{1+2 b_{n} k} a_{n}^{2}+\frac{c_{n}}{1+2 b_{n} k} \\
& =\left(1-\frac{2 b_{n} k}{1+2 b_{n} k}\right) a_{n}^{2}+\frac{c_{n}}{1+2 b_{n} k} .
\end{aligned}
$$

Setting $\alpha_{n}:=2 b_{n} k /\left(1+2 b_{n} k\right)$ and $\beta_{n}:=c_{n} /\left(1+2 b_{n} k\right)$ and by the hypotheses of Lemma 2.3, we get $\alpha_{n} \rightarrow 0$ as $n \rightarrow \infty, \sum_{n} \alpha_{n}=\infty$, and $\lim \sup _{n} \beta_{n} / \alpha_{n}=0$. That is, we reobtain Lemma 2.4 in the case of $c_{n}=0$. 


\section{Main Results}

The ideas of the proofs of our main Theorems take in to account the papers of Chang and Chidume et al. [11, 13, 24].

Theorem 3.1. Let $X$ be a uniformly smooth Banach space, and let $T: X \rightarrow X$ be generalized strongly asymptotically $\phi$-pseudocontractive mapping with fixed point $x^{*}$ and bounded range.

Let $\left\{x_{n}\right\}$ and $\left\{z_{n}\right\}$ be the sequences defined by (1.14) and (1.15), respectively, where $\left\{\alpha_{n}\right\},\left\{\gamma_{n}\right\},\left\{\beta_{n}\right\},\left\{\delta_{n}\right\} \subset[0,1]$ satisfy

(H1) $\lim _{n \rightarrow \infty} \alpha_{n}=\lim _{n \rightarrow \infty} \beta_{n}=\lim _{n \rightarrow \infty} \delta_{n}=0$ and $\gamma_{n}=o\left(\alpha_{n}\right)$,

(H2) $\sum_{n=1}^{\infty} \alpha_{n}=\infty$,

and the sequences $\left\{u_{n}\right\},\left\{v_{n}\right\},\left\{w_{n}\right\}$ are bounded in $X$, then for any initial point $z_{0}, x_{0} \in X$, the following two assertions are equivalent:

(i) the modified Ishikawa iteration sequence with errors (1.14) converges to $x^{*}$;

(ii) the modified Mann iteration sequence with errors (1.15) converges to $x^{*}$.

Proof. First of all, we note that by boundedness of the range of $T$, of the sequences $\left\{w_{n}\right\},\left\{u_{n}\right\}$ and by Lemma 2.4 , it results that $\left\{z_{n}\right\}$ and $\left\{x_{n}\right\}$ are bounded sequences. So, we can set

$$
M=\sup _{n}\left\{\begin{array}{c}
\left\|T^{n} z_{n}-T^{n} y_{n}\right\|,\left\|T^{n} x_{n}-x_{n}\right\|, \\
\left\|T^{n} y_{n}-x_{n}\right\|,\left\|T^{n} z_{n}-z_{n}\right\|, \\
\left\|z_{n}-x_{n}\right\|,\left\|u_{n}-x_{n}\right\|,\left\|v_{n}-x_{n}\right\|, \\
\left\|w_{n}-z_{n}\right\|,\left\|w_{n}-u_{n}\right\|
\end{array}\right\} .
$$

By Lemma 2.2, we have

$$
\begin{aligned}
\left\|z_{n+1}-x_{n+1}\right\|^{2}= & \left\|\left(1-\alpha_{n}-\gamma_{n}\right)\left(z_{n}-x_{n}\right)+\alpha_{n}\left(T^{n} z_{n}-T^{n} y_{n}\right)+\gamma_{n}\left(w_{n}-u_{n}\right)\right\|^{2} \\
\leq & \left(1-\alpha_{n}-\gamma_{n}\right)^{2}\left\|z_{n}-x_{n}\right\|^{2}+2\left\langle\alpha_{n}\left(T^{n} z_{n}-T^{n} y_{n}\right)+\gamma_{n}\left(w_{n}-u_{n}\right), j\left(z_{n+1}-x_{n+1}\right)\right\rangle \\
\leq & \left(1-\alpha_{n}\right)^{2}\left\|z_{n}-x_{n}\right\|^{2}+2 \alpha_{n}\left\langle T^{n} z_{n}-T^{n} y_{n}, j\left(z_{n}-y_{n}\right)\right\rangle \\
& +2 \alpha_{n}\left\langle T^{n} z_{n}-T^{n} y_{n}, j\left(z_{n+1}-x_{n+1}\right)-j\left(z_{n}-y_{n}\right)\right\rangle+2 \gamma_{n}\left\langle w_{n}-u_{n}, j\left(z_{n+1}-x_{n+1}\right)\right\rangle \\
\leq & \left(1-\alpha_{n}\right)^{2}\left\|z_{n}-x_{n}\right\|^{2}+2 \alpha_{n} k_{n}\left\|z_{n}-y_{n}\right\|^{2}-2 \alpha_{n} \phi\left(\left\|z_{n}-y_{n}\right\|\right) \\
& +2 \alpha_{n}\left\|T^{n} z_{n}-T^{n} y_{n}\right\|\left\|j\left(z_{n+1}-x_{n+1}\right)-j\left(z_{n}-y_{n}\right)\right\|+2 \gamma_{n}\left\|w_{n}-u_{n}\right\|\left\|z_{n+1}-x_{n+1}\right\| \\
\leq & \left(1-\alpha_{n}\right)^{2}\left\|z_{n}-x_{n}\right\|^{2}+2 \alpha_{n} k_{n}\left\|z_{n}-y_{n}\right\|^{2}-2 \alpha_{n} \phi\left(\left\|z_{n}-y_{n}\right\|\right) \\
& +2 \alpha_{n} \sigma_{n} M+2 \gamma_{n} M^{2},
\end{aligned}
$$


where $\sigma_{n}=\left\|j\left(z_{n+1}-x_{n+1}\right)-j\left(z_{n}-y_{n}\right)\right\|$. Using (1.14) and (1.15), we have

$$
\begin{aligned}
\left\|\left(z_{n+1}-x_{n+1}\right)-\left(z_{n}-y_{n}\right)\right\| \leq & \left\|x_{n+1}-y_{n}\right\|+\left\|z_{n+1}-z_{n}\right\| \\
= & \left\|\alpha_{n}\left(T^{n} y_{n}-x_{n}\right)+\gamma_{n}\left(u_{n}-x_{n}\right)-\beta_{n}\left(T^{n} x_{n}-x_{n}\right)-\delta_{n}\left(v_{n}-x_{n}\right)\right\| \\
& +\left\|\alpha_{n}\left(T^{n} z_{n}-z_{n}\right)+\gamma_{n}\left(w_{n}-z_{n}\right)\right\| \\
\leq & 2 M\left(\alpha_{n}+\gamma_{n}+\beta_{n}+\delta_{n}\right) \longrightarrow 0, \quad(n \rightarrow \infty) .
\end{aligned}
$$

In view of the uniformly continuity of $j$, we obtain that $\sigma_{n} \rightarrow 0$ as $n \rightarrow \infty$. Furthermore, it follows from the definition of $\left\{y_{n}\right\}$ that for all $n \geq 0$

$$
\begin{aligned}
\left\|z_{n}-y_{n}\right\|^{2} & =\left\|z_{n}-x_{n}+\beta_{n}\left(-T^{n} x_{n}+x_{n}\right)+\delta_{n}\left(-v_{n}+x_{n}\right)\right\|^{2} \\
& \leq\left[\left\|z_{n}-x_{n}\right\|+\beta_{n}\left\|T^{n} x_{n}-x_{n}\right\|+\delta_{n}\left\|v_{n}-x_{n}\right\|\right]^{2} \\
& \leq\left[\left\|z_{n}-x_{n}\right\|+\left(\beta_{n}+\delta_{n}\right) M\right]^{2} \\
& =\left\|z_{n}-x_{n}\right\|^{2}+\left(\beta_{n}+\delta_{n}\right)\left(2\left\|z_{n}-x_{n}\right\| M+\left(\beta_{n}+\delta_{n}\right) M^{2}\right) \\
& \leq\left\|z_{n}-x_{n}\right\|^{2}+3\left(\beta_{n}+\delta_{n}\right) M^{2}, \\
\left\|y_{n}-x_{n}\right\| & \leq \beta_{n}\left\|T^{n} x_{n}-x_{n}\right\|+\delta_{n}\left\|v_{n}-x_{n}\right\| \leq\left(\beta_{n}+\delta_{n}\right) M \longrightarrow 0, \quad(n \rightarrow \infty),
\end{aligned}
$$

so

$$
\begin{aligned}
\left\|z_{n+1}-x_{n+1}\right\| & =\left\|z_{n}-x_{n}-\left(\alpha_{n}+\gamma_{n}\right)\left(z_{n}-x_{n}\right)+\alpha_{n}\left(T^{n} z_{n}-T^{n} y_{n}\right)+\gamma_{n}\left(w_{n}-u_{n}\right)\right\| \\
& \leq\left\|z_{n}-x_{n}\right\|+\left(\alpha_{n}+\gamma_{n}\right)\left\|z_{n}-x_{n}\right\|+\alpha_{n}\left\|T^{n} z_{n}-T^{n} y_{n}\right\|+\gamma_{n}\left\|w_{n}-u_{n}\right\| \\
& \leq\left\|z_{n}-y_{n}\right\|+\left\|y_{n}-x_{n}\right\|+2\left(\alpha_{n}+\gamma_{n}\right) M . \\
& \leq\left\|z_{n}-y_{n}\right\|+\left(\beta_{n}+\delta_{n}\right) M+2\left(\alpha_{n}+\gamma_{n}\right) M .
\end{aligned}
$$

Therefore, we have

$$
\left\|z_{n}-y_{n}\right\| \geq\left\|z_{n+1}-x_{n+1}\right\|-e_{n}
$$

where $e_{n}=\left(\beta_{n}+\delta_{n}\right) M+2\left(\alpha_{n}+\gamma_{n}\right) M$. By (H1), we have that $e_{n} \rightarrow 0$ as $n \rightarrow \infty$. If $\| z_{n+1}-$ $x_{n+1} \|-e_{n} \leq 0$ for an infinite number of indices, we can extract a subsequence such that $\left\|z_{n_{k}}-x_{n_{k}}\right\|-e_{n_{k}-1} \leq 0$. For this subsequence, $\left\|z_{n_{k}}-x_{n_{k}}\right\| \rightarrow 0$, as $k \rightarrow \infty$.

In this case, we can prove that $\left\|z_{n}-x_{n}\right\| \rightarrow 0$, that is, the thesis. 
Journal of Applied Mathematics

Firstly, we note that substituting (3.4) into (3.2), we have

$$
\begin{aligned}
\left\|z_{n+1}-x_{n+1}\right\|^{2} \leq & \left\|z_{n}-x_{n}\right\|^{2}+\alpha_{n}^{2}\left\|z_{n}-x_{n}\right\|^{2}+2 \alpha_{n}\left(k_{n}-1\right)\left\|z_{n}-x_{n}\right\|^{2} \\
& +6 \alpha_{n} k_{n}\left(\beta_{n}+\delta_{n}\right) M^{2}-2 \alpha_{n} \phi\left(\left\|z_{n}-y_{n}\right\|\right)+2 \alpha_{n} \sigma_{n} M+2 \gamma_{n} M^{2} \\
\leq & \left\|z_{n}-x_{n}\right\|^{2}-2 \alpha_{n} \phi\left(\left\|z_{n}-y_{n}\right\|\right)+\alpha_{n}^{2} M^{2}+2 \alpha_{n}\left(k_{n}-1\right) M^{2} \\
& +6 \alpha_{n} \bar{k}\left(\beta_{n}+\delta_{n}\right) M^{2}+2 \alpha_{n} \sigma_{n} M+2 \gamma_{n} M^{2} \\
= & \left\|z_{n}-x_{n}\right\|^{2}-\alpha_{n} \phi\left(\left\|z_{n}-y_{n}\right\|\right)+2 \gamma_{n} M^{2} \\
& -\alpha_{n}\left[\phi\left(\left\|z_{n}-y_{n}\right\|\right)-\alpha_{n} M^{2}-2\left(k_{n}-1\right) M^{2}\right. \\
& \left.-6 \bar{k}\left(\beta_{n}+\delta_{n}\right) M^{2}-2 \sigma_{n} M\right]
\end{aligned}
$$

where $\bar{k}:=\sup _{n}\left(k_{n}\right)$.

Moreover, we observe that

$$
\left\|z_{n_{j}}-y_{n_{j}}\right\| \leq\left\|z_{n_{j}}-x_{n_{j}}\right\|+\left\|y_{n_{j}}-x_{n_{j}}\right\| \longrightarrow 0 \text { as } j \longrightarrow \infty
$$

Thus, for every fixed $\epsilon>0$, there exists $j_{1}$ such that for all $j>j_{1}$

$$
\left\|z_{n_{j}}-y_{n_{j}}\right\|<2 \epsilon \quad\left\|z_{n_{j}}-x_{n_{j}}\right\|<\epsilon .
$$

Since $\left\{\alpha_{n}\right\}_{n},\left\{\left(k_{n}-1\right)\right\}_{n},\left\{\left(\beta_{n}+\delta_{n}\right)\right\}_{n},\left\{\sigma_{n}\right\}_{n}$, and $\left\{\gamma_{n}\right\}_{n}$ are null sequences (and in particular $\left.\gamma_{n}=o\left(\alpha_{n}\right)\right)$, for the previous fixed $\epsilon>0$, there exists an index $N$ such that, for all $n \in N$,

$$
\begin{gathered}
\left|\alpha_{n}\right|<\min \left\{\frac{\epsilon}{16 M^{\prime}}, \frac{\phi(\epsilon / 2)}{8 M^{2}}\right\}, \\
\left|\gamma_{n}\right|<\frac{\epsilon}{16 M^{\prime}}, \quad\left|\frac{\gamma_{n}}{\alpha_{n}}\right|<\frac{\phi(\epsilon / 2)}{4 M^{2}}, \\
\left|k_{n}-1\right|<\frac{\phi(\epsilon / 2)}{16 M^{2}}, \\
\left|\beta_{n}+\delta_{n}\right|<\min \left\{\frac{\epsilon}{4 M}, \frac{\phi(\epsilon / 2)}{48 \bar{k} M^{2}}\right\}, \\
\left|\sigma_{n}\right|<\frac{\phi(\epsilon / 2)}{16 M},
\end{gathered}
$$

for all $n>N$.

Take $n^{*}>\max \left\{N, n_{j_{1}}\right\}$ such that $n^{*}=n_{k}$ for a certain $k$.

We prove, by induction, that $\left\|z_{n^{*}+i}-x_{n^{*}+i}\right\|<\epsilon$, for every $i \in \mathbb{N}$. Let $i=1$. Suppose that $\left\|z_{n^{*}+1}-x_{n^{*}+1}\right\| \geq \epsilon$. 
By (3.6), we have

$$
\begin{aligned}
\epsilon \leq\left\|z_{n^{*}+1}-x_{n^{*}+1}\right\| & \leq\left\|z_{n^{*}}-y_{n^{*}}\right\|+\left(\beta_{n^{*}}+\delta_{n^{*}}\right) M+2\left(\alpha_{n^{*}}+\gamma_{n^{*}}\right) M \\
& \leq\left\|z_{n^{*}}-y_{n^{*}}\right\|+M \frac{\epsilon}{4 M}+2 M\left(\frac{\epsilon}{16 M}+\frac{\epsilon}{16 M}\right) \\
& =\left\|z_{n^{*}}-y_{n^{*}}\right\|+\frac{\epsilon}{4}+\frac{\epsilon}{4}=\left\|z_{n^{*}}-y_{n^{*}}\right\|+\frac{\epsilon}{2} .
\end{aligned}
$$

Thus, $\left\|z_{n^{*}}-y_{n^{*}}\right\| \geq \epsilon / 2$. Since $\phi$ is strictly increasing, $\phi\left(\left\|z_{n^{*}}-y_{n^{*}}\right\|\right) \geq \phi(\epsilon / 2)$.

From (3.8), we obtain that

$$
\begin{aligned}
\left\|z_{n^{*}+1}-x_{n^{*}+1}\right\|^{2}< & \left.\epsilon^{2}-\alpha_{n}^{*}\left(\phi\left\|z_{n^{*}}-y_{n^{*}}\right\|\right)-2 M^{2} \frac{\gamma_{n}^{*}}{\alpha_{n}^{*}}\right) \\
& -\alpha_{n}^{*}\left[\phi\left(\left\|z_{n^{*}}-y_{n^{*}}\right\|\right)-\alpha_{n}^{*} M^{2}-2\left(k_{n}^{*}-1\right) M^{2}-6 \bar{k}\left(\beta_{n}^{*}+\delta_{n}^{*}\right) M^{2}-2 \sigma_{n^{*}} M\right] .
\end{aligned}
$$

One can note that

$$
\alpha_{n}^{*} M^{2}+2\left(k_{n}^{*}-1\right) M^{2}+6 \bar{k}\left(\beta_{n}^{*}+\delta_{n}^{*}\right) M^{2}+2 \sigma_{n^{*}} M \leq \frac{\phi(\epsilon / 2)}{8}+\frac{\phi(\epsilon / 2)}{8}+\frac{\phi(\epsilon / 2)}{8}+\frac{\phi(\epsilon / 2)}{8} .
$$

hence

$$
\phi\left(\left\|z_{n^{*}}-y_{n^{*}}\right\|\right)-\alpha_{n}^{*} M^{2}-2\left(k_{n^{*}}-1\right) M^{2}-6 \bar{k}\left(\beta_{n^{*}}+\delta_{n^{*}}\right) M^{2}-2 \sigma_{n^{*}} M \geq \phi\left(\frac{\epsilon}{2}\right)-\frac{\phi(\epsilon / 2)}{2}>0 .
$$

In the same manner,

$$
\phi\left(\left\|z_{n^{*}}-y_{n^{*}}\right\|\right)-2 M^{2} \frac{\gamma_{n^{*}}}{\alpha_{n^{*}}}>\phi(\epsilon / 2)-\frac{\phi(\epsilon / 2)}{2}>0 .
$$

Thus,

$$
\left\|z_{n^{*}+1}-x_{n^{*}+1}\right\|^{2}<\epsilon^{2}
$$

So we have $\left\|z_{n^{*}+1}-x_{n^{*}+1}\right\|<\epsilon$, which contradicts $\left\|z_{n^{*}+1}-x_{n^{*}+1}\right\| \geq \epsilon$. By the same idea, we can prove that $\left\|z_{n^{*}+2}-x_{n^{*}+2}\right\|<\epsilon$ and then, by inductive step, $\left\|z_{n^{*}+i}-x_{n^{*}+i}\right\| \leq \epsilon$, for all $i$. This is enough to ensure that $\left\|z_{n}-x_{n}\right\| \rightarrow 0$.

If there are only finite indices for which $\left\|z_{n+1}-x_{n+1}\right\|-e_{n} \leq 0$, then definitively $\| z_{n+1}-$ $x_{n+1} \|-e_{n} \geq 0$. By the strict increasing function $\phi$, we have definitively

$$
\phi\left(\left\|z_{n}-y_{n}\right\|\right) \geq \phi\left(\left\|z_{n+1}-x_{n+1}\right\|-e_{n}\right) .
$$


Again substituting (3.4) and (3.18) into (3.2) and simplifying, we have

$$
\begin{aligned}
\left\|z_{n+1}-x_{n+1}\right\|^{2} \leq & \left\|z_{n}-x_{n}\right\|^{2}+\alpha_{n}^{2}\left\|z_{n}-x_{n}\right\|^{2}+2 \alpha_{n}\left(k_{n}-1\right)\left\|z_{n}-x_{n}\right\|^{2} \\
& +6 \alpha_{n} k_{n}\left(\beta_{n}+\delta_{n}\right) M^{2}-2 \alpha_{n} \phi\left(\left\|z_{n+1}-x_{n+1}\right\|-e_{n}\right)+2 \alpha_{n} \sigma_{n} M+2 \gamma_{n} M^{2} \\
\leq & \left\|z_{n}-x_{n}\right\|^{2}-2 \alpha_{n} \phi\left(\left\|z_{n+1}-x_{n+1}\right\|-e_{n}\right)+\alpha_{n}^{2} M^{2}+2 \alpha_{n}\left(k_{n}-1\right) M^{2} \\
& +6 \alpha_{n} k_{n}\left(\beta_{n}+\delta_{n}\right) M^{2}+2 \alpha_{n} \sigma_{n} M+2 \gamma_{n} M^{2} .
\end{aligned}
$$

Suppose that $a_{n}=\left\|z_{n}-x_{n}\right\|, b_{n}=\alpha_{n}$, and $c_{n}=\alpha_{n}^{2} M^{2}+2 \alpha_{n}\left(k_{n}-1\right) M^{2}+6 \alpha_{n} k_{n}\left(\beta_{n}+\delta_{n}\right) M^{2}+$ $2 \alpha_{n} \sigma_{n} M+2 \gamma_{n} M^{2}$. It follows from $\lim _{n \rightarrow \infty} \sigma_{n}=0, \lim _{n \rightarrow \infty} e_{n}=0, \lim _{n \rightarrow \infty} k_{n}=1$, and the hypothesis that we have, $\sum_{n=1}^{\infty} b_{n}=\infty$ and $c_{n}=o\left(b_{n}\right), e_{n} \rightarrow 0$ as $n \rightarrow \infty$. By virtue of Lemma 2.3, we obtain that $\lim _{n \rightarrow \infty} a_{n}=0$. Hence, $\lim _{n \rightarrow \infty}\left\|z_{n}-x_{n}\right\|=0$.

Theorem 3.2. Let $X$ be a uniformly smooth Banach space, and let $T: X \rightarrow X$ be generalized strongly asymptotically $\phi$-pseudocontractive mapping with fixed point $x^{*}$ and bounded range.

Let $\left\{z_{n}\right\}$ and $\left\{z_{n}^{\prime}\right\}$ be the sequences defined by (1.15) and (1.16), respectively, where $\left\{\alpha_{n}\right\},\left\{\gamma_{n}\right\} \subset[0,1]$ are null sequences satisfying

(H1) $\lim _{n \rightarrow \infty} \alpha_{n}=0$ and $\gamma_{n}=o\left(\alpha_{n}\right)$,

(H2) $\sum_{n=1}^{\infty} \alpha_{n}=\infty$,

of Theorem 3.1 and such that $\alpha_{n} k_{n}<1$, for every $n \in \mathbb{N}$.

Suppose moreover that the sequences $\left\{w_{n}\right\},\left\{w_{n}^{\prime}\right\}$ are bounded in $X$, then for any initial point $z_{0}^{\prime}, z_{0} \in X$, the following two assertions are equivalent:

(i) the modified Mann iteration sequence with errors (1.15) converges to the fixed point $x^{*}$,

(ii) the implicit iteration sequence with errors (1.16) converges to the fixed point $x^{*}$.

Proof. As in Theorem 3.1, by the boundedness of the range of $T$ and by Lemma 2.4, one obtains that our schemes are bounded. We define

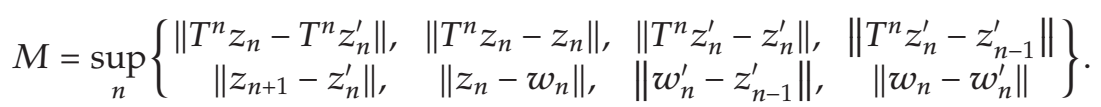

By the iteration schemes (1.15) and (1.16), we have

$$
\begin{aligned}
\left\|z_{n+1}-z_{n}^{\prime}\right\|^{2} \leq & \left\|\left(1-\alpha_{n}-\gamma_{n}\right)\left(z_{n}-z_{n-1}^{\prime}\right)+\alpha_{n}\left(T^{n} z_{n}-T^{n} z_{n}^{\prime}\right)+\gamma_{n}\left(w_{n}-w_{n}^{\prime}\right)\right\|^{2} \\
\leq & \left(1-\alpha_{n}-\gamma_{n}\right)^{2}\left\|z_{n}-z_{n-1}^{\prime}\right\|^{2}+2\left\langle\alpha_{n}\left(T^{n} z_{n}-T^{n} z_{n}^{\prime}\right)+\gamma_{n}\left(w_{n}-w_{n}^{\prime}\right), j\left(z_{n+1}-z_{n}^{\prime}\right)\right\rangle \\
\leq & \left(1-\alpha_{n}\right)^{2}\left\|z_{n}-z_{n-1}^{\prime}\right\|^{2}+2 \alpha_{n}\left\langle T^{n} z_{n}-T^{n} z_{n}^{\prime}, j\left(z_{n}-z_{n}^{\prime}\right)\right\rangle \\
& +2 \alpha_{n}\left\langle T^{n} z_{n}-T^{n} z_{n}^{\prime}, j\left(z_{n+1}-z_{n}^{\prime}\right)-j\left(z_{n}-z_{n}^{\prime}\right)\right\rangle+2 \gamma_{n}\left\|w_{n}-w_{n}^{\prime}\right\|\left\|z_{n+1}-z_{n}^{\prime}\right\| \\
\leq & \left(1-\alpha_{n}\right)^{2}\left\|z_{n}-z_{n-1}^{\prime}\right\|^{2}+2 \alpha_{n} k_{n}\left\|z_{n}-z_{n}^{\prime}\right\|^{2} \\
& -2 \alpha_{n} \phi\left(\left\|z_{n}-z_{n}^{\prime}\right\|\right)+2 \alpha_{n} M \sigma_{n}+2 \gamma_{n} M^{2},
\end{aligned}
$$


where $\sigma_{n}=\left\|j\left(z_{n+1}-z_{n}^{\prime}\right)-j\left(z_{n}-z_{n}^{\prime}\right)\right\|$. By (1.15), we get

$$
\left\|\left(z_{n+1}-z_{n}^{\prime}\right)-\left(z_{n}-z_{n}^{\prime}\right)\right\|=\left\|z_{n+1}-z_{n}\right\|=\left\|\alpha_{n}\left(T^{n} z_{n}-z_{n}\right)+\gamma_{n}\left(w_{n}-z_{n}\right)\right\| \leq\left(\alpha_{n}+\gamma_{n}\right) M .
$$

It follows from (H1) that $\left\|\left(z_{n+1}-z_{n}^{\prime}\right)-\left(z_{n}-z_{n}^{\prime}\right)\right\| \rightarrow 0$ as $n \rightarrow \infty$, which implies that $\sigma_{n} \rightarrow 0$ as $n \rightarrow \infty$. Moreover, for all $n \geq 0$,

$$
\begin{aligned}
\left\|z_{n}-z_{n}^{\prime}\right\|^{2} & \leq\left(\left\|z_{n}-z_{n-1}^{\prime}\right\|+\left\|z_{n-1}^{\prime}-z_{n}^{\prime}\right\|\right)^{2} \\
& \leq\left(\left\|z_{n}-z_{n-1}^{\prime}\right\|+\alpha_{n}\left\|T^{n} z_{n}^{\prime}-z_{n-1}^{\prime}\right\|+\gamma_{n}\left\|w_{n}^{\prime}-z_{n-1}^{\prime}\right\|\right)^{2} \\
& \leq\left[\left\|z_{n}-z_{n-1}^{\prime}\right\|+\left(\alpha_{n}+\gamma_{n}\right) M\right]^{2} \\
& =\left\|z_{n}-z_{n-1}^{\prime}\right\|^{2}+\left(\alpha_{n}+\gamma_{n}\right)\left[2 M\left\|z_{n}-z_{n-1}^{\prime}\right\|+\left(\alpha_{n}+\gamma_{n}\right) M^{2}\right] \\
& \leq\left\|z_{n}-z_{n-1}^{\prime}\right\|^{2}+3\left(\alpha_{n}+\gamma_{n}\right) M^{2} .
\end{aligned}
$$

Again by the boundedness of all components, we have that

$$
\left\|z_{n}^{\prime}-z_{n-1}^{\prime}\right\|=\left\|\alpha_{n}\left(T^{n} z_{n}^{\prime}-z_{n-1}^{\prime}\right)+\gamma_{n}\left(w_{n}^{\prime}-z_{n-1}^{\prime}\right)\right\| \leq\left(\alpha_{n}+\gamma_{n}\right) M,
$$

and so

$$
\begin{aligned}
\left\|z_{n+1}-z_{n}^{\prime}\right\| & =\left\|\left(z_{n}-z_{n}^{\prime}\right)+\left(z_{n}^{\prime}-z_{n-1}^{\prime}\right)-\left(\alpha_{n}+\gamma_{n}\right)\left(z_{n}-z_{n-1}^{\prime}\right)+\alpha_{n}\left(T^{n} z_{n}-T^{n} z_{n}^{\prime}\right)+\gamma_{n}\left(w_{n}-w_{n}^{\prime}\right)\right\| \\
& \leq\left\|z_{n}-z_{n}^{\prime}\right\|+\left\|z_{n}^{\prime}-z_{n-1}^{\prime}\right\|+\left(\alpha_{n}+\gamma_{n}\right)\left\|z_{n}-z_{n-1}^{\prime}\right\|+\alpha_{n}\left\|T^{n} z_{n}-T^{n} z_{n}^{\prime}\right\|+\gamma_{n}\left\|w_{n}-w_{n}^{\prime}\right\| \\
& \leq\left\|z_{n}-z_{n}^{\prime}\right\|+3\left(\alpha_{n}+\gamma_{n}\right) M .
\end{aligned}
$$

Hence, we have that $\left\|z_{n}-z_{n}^{\prime}\right\| \geq\left\|z_{n+1}-z_{n}^{\prime}\right\|-e_{n}$, where $e_{n}=3\left(\alpha_{n}+\gamma_{n}\right) M$. Note that $e_{n} \rightarrow 0$ as $n \rightarrow \infty$. As in proof of Theorem 3.1, we distinguish two cases:

(i) the set of indices for which $\left\|z_{n+1}-z_{n}^{\prime}\right\|-e_{n} \leq 0$ contains infinite terms;

(ii) the set of indices for which $\left\|z_{n+1}-z_{n}^{\prime}\right\|-e_{n} \leq 0$ contains finite terms. 
In the first case, (i) we can extract a subsequence such that $\left\|z_{n_{k}}-z_{n_{k}-1}^{\prime}\right\| \rightarrow 0$, as $k \rightarrow \infty$. Substituting (3.23) in (3.21), we have that

$$
\begin{aligned}
\left\|z_{n+1}-z_{n}^{\prime}\right\|^{2} \leq & \left(1+\alpha_{n}^{2}-2 \alpha_{n}\right)\left\|z_{n}-z_{n-1}^{\prime}\right\|^{2}+2 \alpha_{n} k_{n}\left\|z_{n}-z_{n-1}^{\prime}\right\|^{2}+6\left(\alpha_{n}+\gamma_{n}\right) M^{2} \alpha_{n} k_{n} \\
& -2 \alpha_{n} \phi\left(\left\|z_{n}-z_{n}^{\prime}\right\|\right)+2 \alpha_{n} M \sigma_{n}+2 \gamma_{n} M^{2} \\
\leq & \left\|z_{n}-z_{n-1}^{\prime}\right\|^{2}+\left(\alpha_{n}^{2}+2 \alpha_{n}\left(k_{n}-1\right)\right)\left\|z_{n}-z_{n-1}^{\prime}\right\|^{2}+6\left(\alpha_{n}+\gamma_{n}\right) M^{2} \alpha_{n} \bar{k} \\
& -2 \alpha_{n} \phi\left(\left\|z_{n}-z_{n}^{\prime}\right\|\right)+2 \alpha_{n} M \sigma_{n}+2 \gamma_{n} M^{2} \\
\leq & \left\|z_{n}-z_{n-1}^{\prime}\right\|^{2}-\alpha_{n} \phi\left(\left\|z_{n}-z_{n}^{\prime}\right\|\right)+2 \gamma_{n} M^{2} \\
& -\alpha_{n}\left[\phi\left(\left\|z_{n}-z_{n}^{\prime}\right\|\right)-\alpha_{n} M^{2}-2 M^{2}\left(k_{n}-1\right)-6\left(\alpha_{n}+\gamma_{n}\right) M^{2} \bar{k}-2 M \sigma_{n}\right] \\
= & \left\|z_{n}-z_{n-1}^{\prime}\right\|^{2}-\alpha_{n} \phi\left(\left\|z_{n}-z_{n}^{\prime}\right\|\right)+2 \gamma_{n} M^{2} \\
& -\alpha_{n}\left[\phi\left(\left\|z_{n}-z_{n}^{\prime}\right\|\right)-7 \bar{k} \alpha_{n} M^{2}-2 M^{2}\left(k_{n}-1\right)-6 \gamma_{n} M^{2} \bar{k}-2 M \sigma_{n}\right],
\end{aligned}
$$

where $\bar{k}=\sup _{n} k_{n}$. Again by (3.23), for every $\epsilon>0$, there exists an index $l$ such that if $j>l$,

$$
\left\|z_{n_{j}}-z_{n_{j}-1}^{\prime}\right\|<\epsilon, \quad\left\|z_{n_{j}}-z_{n_{j}}^{\prime}\right\|<2 \epsilon .
$$

By hypotheses on the control sequence, with the same $\epsilon>0$, there exists an index $N$ such that definitively

$$
\begin{gathered}
\left|\alpha_{n}\right|<\min \left\{\frac{\epsilon}{12 M}, \frac{\phi(\epsilon / 2)}{56 M^{2} \bar{k}}\right\} \\
\left|\gamma_{n}\right|<\min \left\{\frac{\epsilon}{12 M}, \frac{\phi(\epsilon / 2)}{48 M^{2} \bar{k}}\right\}, \quad\left|\frac{\gamma_{n}}{\alpha_{n}}\right|<\frac{\phi(\epsilon / 2)}{4 M^{2}}, \\
\left|k_{n}-1\right|<\frac{\phi(\epsilon / 2)}{16 M^{2}}, \\
\left|\sigma_{n}\right|<\frac{\phi(\epsilon / 2)}{16 M} .
\end{gathered}
$$

So take $n^{*}>\max \left\{n_{l}, N\right\}$ with $n^{*}=n_{j}$ for a certain $j$.

We can prove that $\left\|z_{n+1}-z_{n}^{\prime}\right\| \rightarrow 0$ as $n \rightarrow \infty$ proving that, for every $i \geq 0$, the result is $\left\|z_{n^{*}+i}-z_{n^{*}+i-1}^{\prime}\right\|<\epsilon$.

Let $i=1$. If we suppose that $\left\|z_{n^{*}+1}-z_{n^{*}}^{\prime}\right\| \geq \epsilon$, it results that

$$
\epsilon \leq\left\|z_{n^{*}+1}-z_{n^{*}}^{\prime}\right\| \leq\left\|z_{n^{*}}-z_{n^{*}}^{\prime}\right\|+3\left(\alpha_{n^{*}}+\gamma_{n^{*}}\right) M<\left\|z_{n^{*}}-z_{n^{*}}^{\prime}\right\|+\frac{\epsilon}{2},
$$

so $\left\|z_{n^{*}}-z_{n^{*}}^{\prime}\right\|>\epsilon / 2$. In consequence of this, $\phi\left(\left\|z_{n^{*}}-z_{n^{*}}^{\prime}\right\|\right)>\phi(\epsilon / 2)$. 
In (3.26), we note that

$$
\begin{aligned}
7 \bar{k} \alpha_{n^{*}} & M^{2}+2 M^{2}\left(k_{n^{*}}-1\right)+6 \gamma_{n^{*}} M^{2} \bar{k}+2 M \sigma_{n^{*}} \\
& \leq 7 \bar{k} M^{2} \frac{\phi(\epsilon / 2)}{56 M^{2} \bar{k}}+2 M^{2} \frac{\phi(\epsilon / 2)}{16 M^{2}}+6 M^{2} \bar{k} \frac{\phi(\epsilon / 2)}{48 M^{2} \bar{k}}+2 M \frac{\phi(\epsilon / 2)}{16 M} \\
& =\frac{\phi(\epsilon / 2)}{8} 4=\frac{\phi(\epsilon / 2)}{2}
\end{aligned}
$$

so

$$
\phi\left(\left\|z_{n^{*}}-z_{n^{*}}^{\prime}\right\|\right)-7 \bar{k} \alpha_{n^{*}} M^{2}-2 M^{2}\left(k_{n^{*}}-1\right)-6 \gamma_{n^{*}} M^{2} \bar{k}-2 M \sigma_{n^{*}} \geq \frac{\phi(\epsilon / 2)}{2} .
$$

hence in (3.26) remains

$$
\left\|z_{n^{*}+1}-z_{n^{*}}^{\prime}\right\|^{2} \leq \epsilon^{2}-\alpha_{n} \phi\left(\left\|z_{n}-z_{n}^{\prime}\right\|\right)+2 \gamma_{n} M^{2}<\epsilon^{2}
$$

as in Theorem 3.1. This is a contradiction. By the same idea, and using the inductive hypothesis, we obtain that $\left\|z_{n^{*}+i}-z_{n^{*}+i-1}^{\prime}\right\|<\epsilon$, for every $i \geq 0$. This ensures that $\left\|z_{n+1}-z_{n}^{\prime}\right\| \rightarrow$ 0 . In the second case (ii), definitively, $\left\|z_{n+1}-z_{n}^{\prime}\right\|-e_{n} \geq 0$, then from the strictly increasing function $\phi$, we have

$$
\phi\left(\left\|z_{n}-z_{n}^{\prime}\right\|\right) \geq \phi\left(\left\|z_{n+1}-z_{n}^{\prime}\right\|-e_{n}\right) .
$$

Substituting (3.33) and (3.23) into (3.21) and simplifying, we have

$$
\begin{aligned}
\left\|z_{n+1}-z_{n}^{\prime}\right\|^{2} \leq & \left\|z_{n}-z_{n-1}^{\prime}\right\|^{2}+\alpha_{n}^{2}\left\|z_{n}-z_{n-1}^{\prime}\right\|^{2}+2 \alpha_{n}\left(k_{n}-1\right)\left\|z_{n}-z_{n-1}^{\prime}\right\|^{2} \\
& +6 \alpha_{n} k_{n}\left(\alpha_{n}+\gamma_{n}\right) M^{2}-2 \alpha_{n} \phi\left(\left\|z_{n+1}-z_{n}^{\prime}\right\|-e_{n}\right)+2 \alpha_{n} \sigma_{n} M+2 \gamma_{n} M^{2} \\
\leq & \left\|z_{n}-z_{n-1}^{\prime}\right\|^{2}-2 \alpha_{n} \phi\left(\left\|z_{n+1}-z_{n}^{\prime}\right\|-e_{n}\right)+\alpha_{n}^{2} M^{2} \\
& +2 \alpha_{n}\left(k_{n}-1\right) M^{2}+6 \alpha_{n} k_{n}\left(\alpha_{n}+\gamma_{n}\right) M^{2}+2 \alpha_{n} \sigma_{n} M+2 \gamma_{n} M^{2} .
\end{aligned}
$$

By virtue of Lemma 2.3, we obtain that $\lim _{n \rightarrow \infty}\left\|z_{n}-z_{n-1}^{\prime}\right\|=0$.

Theorem 3.3. Let X be a uniformly smooth Banach space, and let $T: X \rightarrow X$ be generalized strongly asymptotically $\phi$-pseudocontractive mapping with fixed point $x^{*}$ and bounded range.

Let $\left\{z_{n}\right\}_{n}$ be the sequences defined by (1.15) where $\left\{\alpha_{n}\right\}_{n},\left\{\gamma_{n}\right\}_{n} \subset[0,1]$ satisfy

(i) $\lim _{n \rightarrow \infty} \alpha_{n}=\lim _{n \rightarrow \infty} \gamma_{n}=0$,

(ii) $\sum_{n=1}^{\infty} \alpha_{n}=\infty, \sum_{n=1}^{\infty} \gamma_{n}<\infty$.

and the sequence $\left\{w_{n}\right\}_{n}$ is bounded on $X$, then for any initial point $z_{0} \in X$, the sequence $\left\{z_{n}\right\}_{n}$ strongly converges to $x^{*}$. 
Proof. Firstly, we observe that, by the boundedness of the range of $T$, of the sequence $\left\{w_{n}\right\}_{n}$, and by Lemma 2.4, we have that $\left\{z_{n}\right\}_{n}$ is bounded.

By Lemma 2.2, we observe that

$$
\begin{aligned}
\left\|z_{n+1}-x^{*}\right\|^{2} \leq & \left(1-\alpha_{n}-\gamma_{n}\right)^{2}\left\|z_{n}-x^{*}\right\|^{2}+2 \alpha_{n}\left\langle T^{n} z_{n}-x^{*}, j\left(z_{n+1}-x^{*}\right\rangle\right. \\
& +2 \gamma_{n}\left\langle w_{n}-x^{*}, j\left(z_{n+1}-x^{*}\right)\right\rangle \\
\leq & \left(1-\alpha_{n}\right)^{2}\left\|z_{n}-x^{*}\right\|^{2}+2 \alpha_{n}\left\langle T^{n} z_{n}-x^{*}, j\left(z_{n+1}-x^{*}\right)-j\left(z_{n}-x^{*}\right)\right\rangle \\
& +2 \alpha_{n}\left\langle T^{n} z_{n}-x^{*}, j\left(z_{n}-x^{*}\right)\right\rangle+2 \gamma_{n}\left\langle w_{n}-x^{*}, j\left(z_{n+1}-x^{*}\right)\right\rangle \\
\leq & \left(1-\alpha_{n}\right)^{2}\left\|z_{n}-x^{*}\right\|^{2}+2 \alpha_{n}\left\langle T^{n} n z_{n}-x^{*}, j\left(z_{n+1}-x^{*}\right)-j\left(z_{n}-x^{*}\right)\right\rangle \\
& +2 \alpha_{n} k_{n}\left\|z_{n}-x^{*}\right\|^{2}-2 \alpha_{n} \phi\left(\left\|z_{n}-x^{*}\right\|\right)+2 \gamma_{n}\left\langle w_{n}-x^{*}, j\left(z_{n+1}-x^{*}\right)\right\rangle \\
= & \left(1+\alpha_{n}^{2}-2 \alpha_{n}\right)\left\|z_{n}-x^{*}\right\|^{2}+2 \alpha_{n}\left\langle T^{n} z_{n}-x^{*}, j\left(z_{n+1}-x^{*}\right)-j\left(z_{n}-x^{*}\right)\right\rangle \\
& +2 \alpha_{n} k_{n}\left\|z_{n}-x^{*}\right\|^{2}-2 \alpha_{n} \phi\left(\left\|z_{n}-x^{*}\right\|\right)+2 \gamma_{n}\left\langle w_{n}-x^{*}, j\left(z_{n+1}-x^{*}\right)\right\rangle \\
= & \left\|z_{n}-x^{*}\right\|^{2}+\left(\alpha_{n}^{2}-2 \alpha_{n}+2 \alpha_{n} k_{n}\right)\left\|z_{n}-x^{*}\right\|^{2}-2 \alpha_{n} \phi\left(\left\|z_{n}-x^{*}\right\|\right)+2 \alpha_{n} \mu_{n} \\
& +2 \gamma_{n}\left\langle w_{n}-x^{*}, j\left(z_{n+1}-x^{*}\right)\right\rangle,
\end{aligned}
$$

where $\mu_{n}:=\left\langle T^{n} z_{n}-x^{*}, j\left(z_{n+1}-x^{*}\right)-j\left(z_{n}-x^{*}\right)\right\rangle$. Let

$$
M:=\max \left\{\sup _{n}\left\|z_{n}-x^{*}\right\|, \sup _{n}\left\|T^{n} z_{n}-x^{*}\right\|, \sup _{n}\left\|w_{n}-x^{*}\right\|, \sup _{n}\left\langle w_{n}-x^{*}, j\left(z_{n+1}-x^{*}\right)\right\rangle\right\} .
$$

We have

$$
\begin{aligned}
\left\|z_{n+1}-x^{*}\right\|^{2} \leq & \left\|z_{n}-x^{*}\right\|^{2}+\left(\alpha_{n}^{2}+2 \alpha_{n}\left(k_{n}-1\right)\right) M-2 \alpha_{n} \phi\left(\left\|z_{n}-x^{*}\right\|\right)+2 \alpha_{n} \mu_{n}+2 \gamma_{n} M \\
= & \left\|z_{n}-x^{*}\right\|^{2}-\alpha_{n} \phi\left(\left\|z_{n}-x^{*}\right\|\right)+2 \gamma_{n} M \\
& -\alpha_{n}\left[\phi\left(\left\|z_{n}-x^{*}\right\|\right)-2 \mu_{n}-\left(\alpha_{n}+2\left(k_{n}-1\right)\right) M\right],
\end{aligned}
$$

so we can observe that

(1) $\mu_{n} \rightarrow 0$ as $n \rightarrow \infty$. Indeed from the inequality

$$
\left|\left\|z_{n+1}-x^{*}\right\|-\left\|z_{n}-x^{*}\right\|\right| \leq\left\|z_{n+1}-z_{n}\right\| \leq\left(\alpha_{n}+\gamma_{n}\right) M \longrightarrow 0, \quad \text { as } n \longrightarrow \infty,
$$

and since $j$ is norm to norm uniformly continuous, then $j\left(\left\|z_{n+1}-x^{*}\right\|\right)-j\left(\| z_{n}-\right.$ $\left.x^{*} \|\right) \rightarrow 0$, as $n \rightarrow \infty$, 
(2) $\inf _{n}\left(\left\|z_{n}-x^{*}\right\|\right)=0$. Indeed, if we supposed that $\sigma:=\inf _{n}\left(\left\|z_{n}-x^{*}\right\|\right)>0$, by the monotonicity of $\phi$,

$$
\phi\left(\left\|z_{n}-x^{*}\right\|\right) \geq \phi(\sigma)>0 .
$$

Thus, by (1) and by the hypotheses on $\alpha_{n}$ and $k_{n}$, the value $-\alpha_{n}\left[\phi\left(\left\|z_{n}-x^{*}\right\|\right)-2 \mu_{n}-\right.$ $\left.\left(\alpha_{n}+2\left(k_{n}-1\right)\right) M\right]$ is definitively negative. In this case, we conclude that there exists $N>0$ such that for every $n>N$,

$$
\begin{aligned}
\left\|z_{n+1}-x^{*}\right\|^{2} & \leq\left\|z_{n}-x^{*}\right\|^{2}-\alpha_{n} \phi\left(\left\|z_{n}-x^{*}\right\|\right)+2 \gamma_{n} M \\
& \leq\left\|z_{n}-x^{*}\right\|^{2}-\alpha_{n} \phi(\sigma)+2 \gamma_{n} M,
\end{aligned}
$$

and so

$$
\alpha_{n} \phi(\sigma) \leq\left\|z_{n}-x^{*}\right\|^{2}-\left\|z_{n+1}-x^{*}\right\|^{2}+2 \gamma_{n} M \quad \forall n>N .
$$

In the same way we obtain that

$$
\begin{gathered}
\phi(\sigma) \sum_{i=N}^{m} \alpha_{i} \leq \sum_{i=N}^{m}\left[\left\|z_{i}-x^{*}\right\|^{2}-\left\|z_{i+1}-x^{*}\right\|^{2}\right]+2 \sum_{i=N}^{m} \gamma_{i} M \\
=\left\|z_{N}-x^{*}\right\|^{2}-\left\|z_{m}-x^{*}\right\|^{2}+2 M \sum_{i=N}^{m} \gamma_{i} .
\end{gathered}
$$

By the hypotheses $\sum_{n} \gamma_{n}<\infty$ and $\sum_{n} \alpha_{n}=\infty$, the previous is a contradiction, and it follows that $\inf _{n}\left(\left\|z_{n}-x^{*}\right\|\right)=0$.

Then, there exists a subsequence $\left\{z_{n_{k}}\right\}_{k}$ of $\left\{z_{n}\right\}_{n}$ that strongly converges to $x^{*}$. This implies that for every $\epsilon>0$, there exists an index $n_{k(\epsilon)}$ such that, for all $j \geq n_{k(\epsilon)},\left\|z_{n_{j}}-x^{*}\right\|<\epsilon$.

Now, we will prove that the sequence $\left\{z_{n}\right\}_{n}$ converges to $x^{*}$. Since the sequences in (3.37) are null sequences and $\sum_{n} \gamma_{n}<\infty$, but $\sum_{n} \alpha_{n}=\infty$, then, for every $\epsilon>0$, there exists an index $\bar{n}(\epsilon)$ such that for all $n \geq \bar{n}(\epsilon)$, it results that

$$
\begin{gathered}
\left|\alpha_{n}\right|<\frac{1}{4 M} \min \left\{\epsilon, \phi\left(\frac{\epsilon}{2}\right)\right\}, \\
\left|\gamma_{n}\right|<\frac{\epsilon}{4 M}, \quad\left|\frac{\gamma_{n}}{\alpha_{n}}\right|<\frac{\phi(\epsilon / 2)}{4 M}, \\
\left|k_{n}-1\right|<\frac{\phi(\epsilon / 2)}{8 M} \\
\left|\mu_{n}\right|<\frac{\phi(\epsilon / 2)}{8}
\end{gathered}
$$


So, fixing $\epsilon>0$, let $n^{*}>\max \left(n_{k(\epsilon)}, \bar{n}(\epsilon)\right)$ with $n^{*}=n_{j}$ for a certain $n_{j}$. We will prove, by induction, that $\left\|z_{n^{*}+i}-x^{*}\right\|<\epsilon$ for every $i \in \mathbb{N}$. Let $i=1$. If not, it results that $\left\|z_{n^{*}+1}-x^{*}\right\| \geq \epsilon$. Thus,

$$
\begin{aligned}
\epsilon & \leq\left\|z_{n^{*}+1}-x^{*}\right\| \leq\left\|z_{n^{*}}-x^{*}\right\|+\alpha_{n^{*}} M+\gamma_{n^{*}} M, \\
& <\left\|z_{n^{*}}-x^{*}\right\|+\frac{\epsilon}{4 M} M+\frac{\epsilon}{4 M} M=\left\|z_{n^{*}}-x^{*}\right\|+\frac{\epsilon}{2},
\end{aligned}
$$

that is, $\left\|z_{n^{*}}-x^{*}\right\|>\epsilon / 2$. By the strict increasing of $\phi, \phi\left(\left\|z_{n^{*}}-x^{*}\right\|\right)>\phi(\epsilon / 2)$.

By (3.37), it results that

$$
\begin{aligned}
\left\|z_{n^{*}+1}-x^{*}\right\|^{2}< & \epsilon^{2}-\alpha_{n^{*}}\left(\phi\left(\left\|z_{n^{*}}-x^{*}\right\|\right)-2 M \frac{\gamma_{n^{*}}}{\alpha_{n^{*}}}\right) \\
& -\alpha_{n^{*}}\left[\phi\left(\left\|z_{n^{*}}-x^{*}\right\|\right)-2 \mu_{n^{*}}-\left(\alpha_{n^{*}}+2\left(k_{n^{*}}-1\right)\right) M\right] .
\end{aligned}
$$

We can note that

$$
2 \mu_{n^{*}}+\left(\alpha_{n^{*}}+2\left(k_{n^{*}}-1\right)\right) M \leq \frac{\phi(\epsilon / 2)}{4}+\left(\frac{\phi(\epsilon / 2)}{4 M}+\frac{\phi(\epsilon / 2)}{4 M}\right) M
$$

so

$$
\phi\left(\left\|z_{n^{*}}-x^{*}\right\|\right)-2 \mu_{n^{*}}-\left(\alpha_{n^{*}}+2\left(k_{n^{*}}-1\right)\right) M>\phi(\epsilon / 2)-\frac{3 \phi(\epsilon / 2)}{4}>0 .
$$

Moreover, $\phi\left(\left\|z_{n^{*}}-x^{*}\right\|\right)-2 M \gamma_{n^{*}} / \alpha_{n^{*}}>\phi(\epsilon / 2) / 2>0$, so it results that

$$
\left\|z_{n^{*}+1}-x^{*}\right\|^{2}<\epsilon^{2} .
$$

This is a contradiction. Thus, $\left\|z_{n^{*}+1}-x^{*}\right\|<\epsilon$.

In the same manner, by induction, one obtains that, for every $i \geq 1,\left\|z_{n^{*}+i}-x^{*}\right\|<\epsilon$. So $\left\|z_{n}-x^{*}\right\| \rightarrow 0$.

Corollary 3.4. Let $X$ be a uniformly smooth Banach space, and let $T: X \rightarrow X$ be generalized strongly asymptotically $\phi$-pseudocontractive mapping with bounded range and fixed point $x^{*}$. The sequences $\left\{x_{n}\right\}_{n},\left\{z_{n}\right\}_{n}$, and $\left\{z_{n}^{\prime}\right\}_{n}$ are defined by (1.14), (1.15), and (1.16), respectively, where the sequences $\left\{\alpha_{n}\right\}_{n},\left\{\beta_{n}\right\}_{n},\left\{\gamma_{n}\right\}_{n},\left\{\delta_{n}\right\}_{n} \subset[0,1]$ satisfy

(i) $\lim _{n \rightarrow \infty} \alpha_{n}=\lim _{n \rightarrow \infty} \beta_{n}=\lim _{n \rightarrow \infty} \delta_{n}=0$,

(ii) $\sum_{n=1}^{\infty} \alpha_{n}=\infty, \sum_{n=1}^{\infty} \gamma_{n}<\infty$,

and the sequences $\left\{u_{n}\right\}_{n},\left\{v_{n}\right\}_{n},\left\{w_{n}\right\}_{n}$, and $\left\{w_{n}^{\prime}\right\}_{n}$ are bounded in $X$. Then for any initial point $x_{0}, z_{0}, z_{0}^{\prime} \in X$, the following two assertions are equivalent and true:

(i) the modified Ishikawa iteration sequence with errors (1.14) converges to the fixed point $x^{*}$;

(ii) the modified Mann iteration sequence with errors (1.15) converges to the fixed point $x^{*}$;

(iii) the implicit iteration sequence with errors (1.16) converges to the fixed point $x^{*}$. 


\section{References}

[1] F. E. Browder, "Nonlinear mappings of nonexpansive and accretive type in Banach spaces," Bulletin of the American Mathematical Society, vol. 73, pp. 875-882, 1967.

[2] T. Kato, "Nonlinear semigroups and evolution equations," Journal of the Mathematical Society of Japan, vol. 19, pp. 508-520, 1967.

[3] C. Chidume, Geometric properties of Banach spaces and nonlinear iterations, vol. 1965 of Lecture Notes in Mathematics, Springer, London, UK, 2009.

[4] K. Goebel and W. A. Kirk, "A fixed point theorem for asymptotically nonexpansive mappings," Proceedings of the American Mathematical Society, vol. 35, pp. 171-174, 1972.

[5] K. Deimling, "Zeros of accretive operators," Manuscripta Mathematica, vol. 13, pp. 365-374, 1974.

[6] M. O. Osilike, "Iterative solution of nonlinear equations of the $\phi$-strongly accretive type," Journal of Mathematical Analysis and Applications, vol. 200, no. 2, pp. 259-271, 1996.

[7] C. H. Xiang, "Fixed point theorem for generalized $\phi$-pseudocontractive mappings," Nonlinear Analysis, vol. 70, no. 6, pp. 2277-2279, 2009.

[8] J. Schu, "Iterative construction of fixed points of asymptotically nonexpansive mappings," Journal of Mathematical Analysis and Applications, vol. 158, no. 2, pp. 407-413, 1991.

[9] E. U. Ofoedu, "Strong convergence theorem for uniformly L-Lipschitzian asymptotically pseudocontractive mapping in real Banach space," Journal of Mathematical Analysis and Applications, vol. 321, no. 2, pp. 722-728, 2006.

[10] H. Zhou, "Demiclosedness principle with applications for asymptotically pseudo-contractions in Hilbert spaces," Nonlinear Analysis, vol. 70, no. 9, pp. 3140-3145, 2009.

[11] C. E. Chidume and M. O. Osilike, "Equilibrium points for a system involving $m$-accretive operators," Proceedings of the Edinburgh Mathematical Society. Series II, vol. 44, no. 1, pp. 187-199, 2001.

[12] C. E. Chidume and H. Zegeye, "Approximate fixed point sequences and convergence theorems for Lipschitz pseudocontractive maps," Proceedings of the American Mathematical Society, vol. 132, no. 3, pp. 831-840, 2004.

[13] S. S. Chang, "Some results for asymptotically pseudo-contractive mappings and asymptotically nonexpansive mappings," Proceedings of the American Mathematical Society, vol. 129, no. 3, pp. 845853, 2001.

[14] K. Deimling, Nonlinear Functional Analysis, Springer, Berlin, Germany, 1985.

[15] S. S. Chang, K. K. Tan, H. W. J. Lee, and C. K. Chan, “On the convergence of implicit iteration process with error for a finite family of asymptotically nonexpansive mappings," Journal of Mathematical Analysis and Applications, vol. 313, no. 1, pp. 273-283, 2006.

[16] F. Gu, "The new composite implicit iterative process with errors for common fixed points of a finite family of strictly pseudocontractive mappings," Journal of Mathematical Analysis and Applications, vol. 329, no. 2, pp. 766-776, 2007.

[17] Z. Huang and F. Bu, "The equivalence between the convergence of Ishikawa and Mann iterations with errors for strongly successively pseudocontractive mappings without Lipschitzian assumption," Journal of Mathematical Analysis and Applications, vol. 325, no. 1, pp. 586-594, 2007.

[18] L. S. Liu, "Ishikawa and Mann iterative process with errors for nonlinear strongly accretive mappings in Banach spaces," Journal of Mathematical Analysis and Applications, vol. 194, no. 1, pp. 114-125, 1995.

[19] B. E. Rhoades and S. M. Soltuz, "The equivalence between the convergences of Ishikawa and Mann iterations for an asymptotically nonexpansive in the intermediate sense and strongly successively pseudocontractive maps," Journal of Mathematical Analysis and Applications, vol. 289, no. 1, pp. 266$278,2004$.

[20] B. E. Rhoades and S. M. Soltuz, "The equivalence between Mann-Ishikawa iterations and multistep iteration," Nonlinear Analysis, vol. 58, no. 1-2, pp. 219-228, 2004.

[21] Z. Huang, "Equivalence theorems of the convergence between Ishikawa and Mann iterations with errors for generalized strongly successively $\phi$-pseudocontractive mappings without Lipschitzian assumptions," Journal of Mathematical Analysis and Applications, vol. 329, no. 2, pp. 935-947, 2007.

[22] Y. Xu, "Ishikawa and Mann iterative processes with errors for nonlinear strongly accretive operator equations," Journal of Mathematical Analysis and Applications, vol. 224, no. 1, pp. 91-101, 1998.

[23] P.-E. Maingé, "Approximation methods for common fixed points of nonexpansive mappings in Hilbert spaces," Journal of Mathematical Analysis and Applications, vol. 325, no. 1, pp. 469-479, 2007.

[24] C. E. Chidume and C. O. Chidume, "Convergence theorem for zeros of generalized lipschitz generalized phi-quasi-accretive operators," Proceedings of the American Mathematical Society, vol. 134, no. 1, pp. 243-251, 2006. 


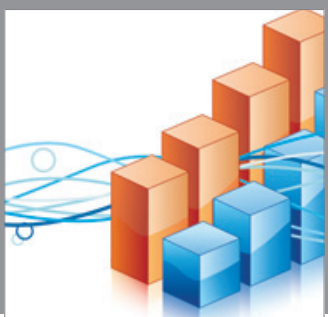

Advances in

Operations Research

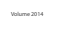

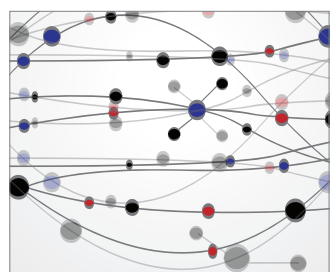

\section{The Scientific} World Journal
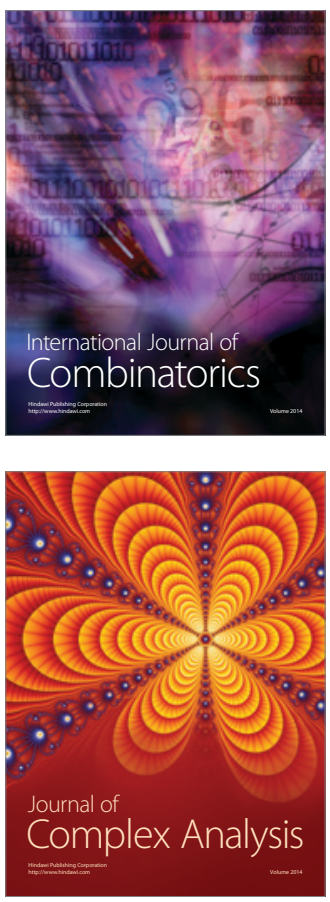

International Journal of

Mathematics and

Mathematical

Sciences
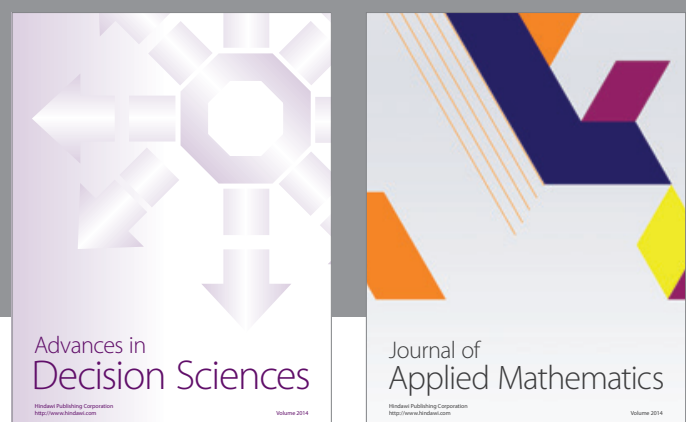

Journal of

Applied Mathematics
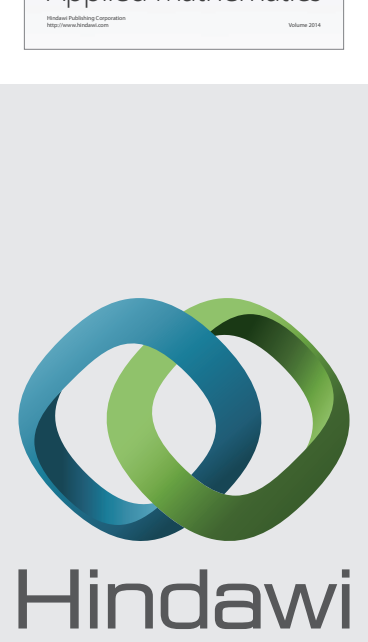

Submit your manuscripts at http://www.hindawi.com
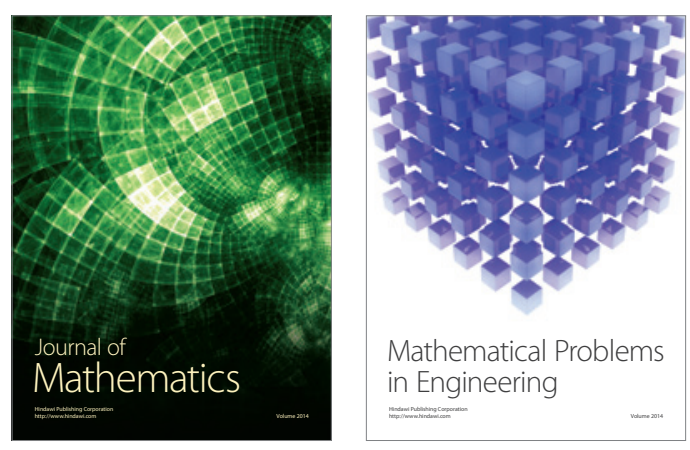

Mathematical Problems in Engineering
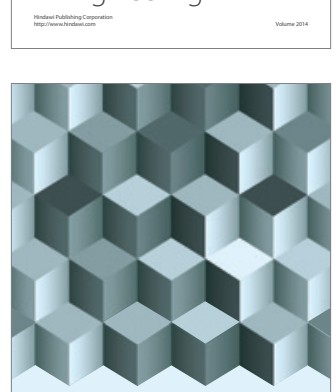

Journal of

Function Spaces
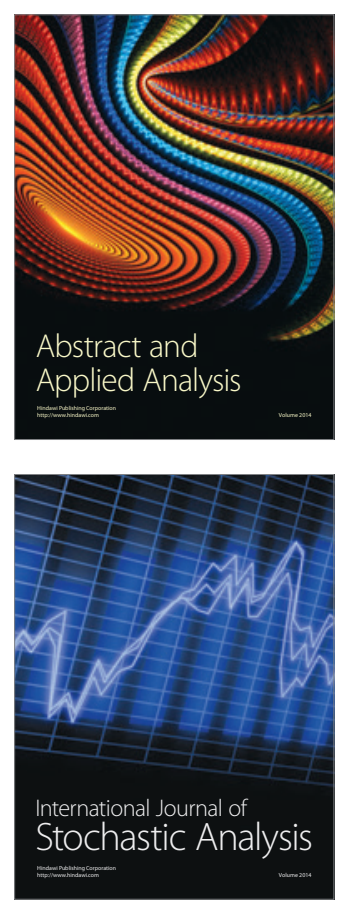

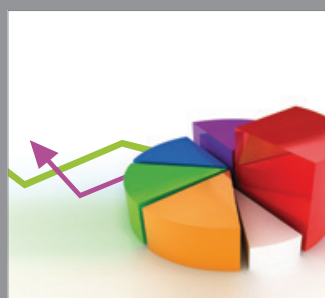

ournal of

Probability and Statistics

Promensencen
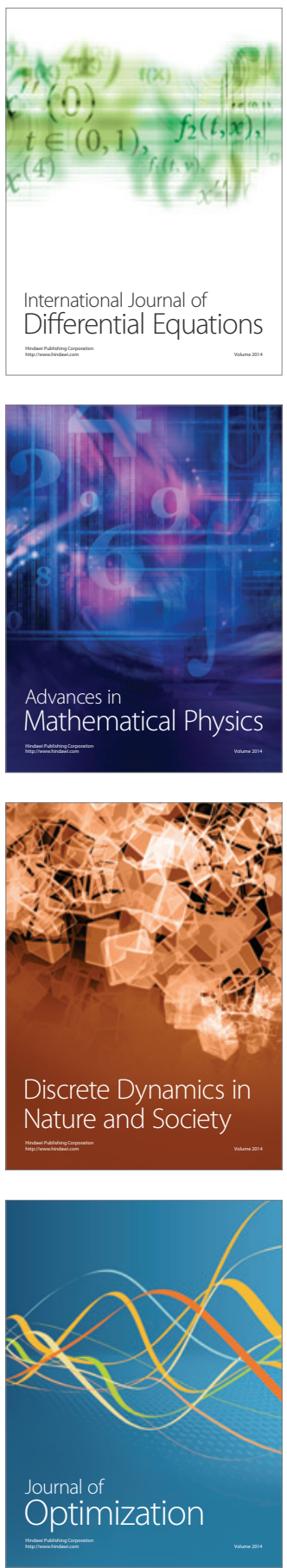